\title{
El discurso ausente: el caso del cambio climático
}

\author{
Javier Callejo Gallego
}

UNED / Universidad Complutense de Madrid. TRANSOC

mcallejo@poli.uned.es

\section{Resumen}

Partiendo de una investigación empírica que incluye entre sus objetivos la observación de la preocupación de la sociedad española por el cambio climático, el presente trabajo indaga en el potencial heurístico que tiene el silencio recogido sobre este fenómeno desde las técnicas de investigación cualitativas. Según los resultados de encuestas con cuestionario estandarizado, el cambio climático preocupa a los españoles. Según los grupos de discusión, no aparece entre las preocupaciones. Esto exige una reflexión sobre lo siguiente: a) qué es un discurso social, frente a otro tipo de materiales lingüísticos; b) cuándo, en lugar de tal discurso, se recoge silencio; c) las condiciones de observación empírica para la recogida de tal silencio.

Palabras clave: métodos mixtos; metodología; investigación social; discurso social; preocupación social; sociología medioambiental

\section{Abstract. The absent discours: the climate change case}

From an empirical investigation that includes observation of the Spanish society's concern about climate change among its objectives, the present work investigates the heuristic potential of the silence collected on this phenomenon from qualitative social research methods. According to own survey results Spaniards are very concerned about climate change. But according to the discussion groups results, climate change does not appear among the concerns. This requires a reflection on: a) what is a social discourse, compared to other types of linguistic materials; b) when silence is collected instead of such as a social discourse; c) empirical observation conditions for the collection of such silence.

Keywords: mixed methods; methodology; social research; social discourse; social concern; environmental sociology 


\section{Sumario}
1. Introducción
5. Conclusiones tentativas
2. Contextos concretos de observación
Agradecimientos
3. Resultados
Financiación
4. Hacia la interpretación del no hablar y
Referencias bibliográficas las condiciones del discurso social

\section{Introducción}

En un estimulante texto, Zerubavel (2006) se pregunta cómo se puede callar una evidencia como la existencia de un elefante en la habitación. El presente trabajo expone la ausencia de referencias a un fenómeno de la importancia del cambio climático, ${ }^{1}$ cuando se cuestiona a los informantes sobre el futuro y las preocupaciones que lo pueblan. Esta ausencia nos lleva a reflexionar sobre las condiciones que ha de tener un habla para ser considerada un discurso de la sociedad, entre la gente, en sus mundos de vida. Por otro lado, esta ausencia contrasta con los resultados —o puntos de partida — de un abundante número de investigaciones fundamentadas en el análisis de discursos medioambientales de distintas características. En esta introducción, se destacan las líneas más relevantes:

a) Discursos expertos y semiexpertos. ${ }^{2}$

b) Discursos sobre los miedos presentes en los discursos públicos con relación al cambio climático, como hace el trabajo de Hulme (2008).

c) Discursos de actores relevantes en el fenómeno, como los movimientos ecologistas (Luke, 1995; 1999; Dickens, 1992; Fill y Mühlhäusler, 2001; Miranda, 2007), la política (O’Riordan y Jäger, 1996) o las corporaciones (Howlet y Raglon, 2001).

d) Discursos de los medios de comunicación, donde destacan los estudios de Boykoff (2008; 2013), Boykoff y Boykoff (2004), Boykoff y Roberts (2007) y Carvalho (2005; 2010), entre una ingente cantidad (entre otros, Antilla, 2005; Greer y Bruno, 1996; Müslhäusler, 2003; Nisbet, 2009; Szerszynski, 2010). Análisis del discurso de las distintas unidades y géneros (reportajes, artículos de opinión, etcétera) aparecidos en los medios de

1. Se utiliza la forma cambio climático, frente a calentamiento global o efecto invernadero, al haberse impuesto en la mayor parte de los documentos institucionales.

2. Un ejemplo de investigaciones sobre discursos medioambientales expertos está en el estudio que Harré et al. (1999) denominan Greenspeak tomando como corpus: conferencias en el Linacre College (Oxford); informes de la Conferencia de Río de Janeiro de 1992; documentos y manifestaciones del Partido Ecologista, y artículos de revistas de alta divulgación científica, como Scientific American. Más recientemente, Ramos (2018) analiza los discursos expertos desde la sociología del tiempo y las distintas temporalidades futuras que presentan. Desde la gobernanza de los conflictos medioambientales a través del diálogo entre expertos y ciudadanos («legos»), véase Prades et al. (2015). 
comunicación de notable audiencia (programas de televisión con cobertura nacional, diarios de tirada nacional que buscan cubrir un amplio espectro ideológico. En nuestra investigación, el objetivo es distinto a la cobertura mediática de este tema, tal como hacen, para el caso español, Fernández Reyes et al. (2015), Vicente-Mariño, Vicente-Torrico (2014) o Quesada Pérez et al. (2015). Nuestro esfuerzo estaba destinado a aproximarnos a la específica relación de la sociedad española con el tema.

e) Los estudios que captan la opinión de la sociedad en general, de los denominados legos desde la sociología de la ciencia y la tecnología, por oposición a los actores cualificados (Lofstedt, 1991; Wynne, 1994; 1996; Poortinga y Pigdeon, 2003; Poortinga et al., 2006; Kempton et al., 1995; Lash et al., 1996; Dunlap, 1998; Wibeck et al., 2015; Bostrom et al., 1991; Smith, 2007; Brechin, 2008). En España, también han sido numerosos los estudios que se han enfrentado a la opinión pública sobre los problemas medioambientales y sus políticas. Entre los muchos que existen, se destacan los de Sala et al. (2008), Moyano et al. (2009), Santiago (2006), sobre el análisis material de encuestas en distintos contextos, o Oltra et al. (2015), sobre la percepción de riesgos de la contaminación ${ }^{3}$.

Hay que poner de manifiesto que la mayor parte de los estudios centrados en los discursos institucionales entienden que son recibidos por los legos y, en buena medida, reproducidos en forma de opinión pública — esta estructura de la línea de estudios se denomina agenda setting. Al menos, no se cuestiona tal recepción (Pettenger, 2007), entendida como el uso, en sus mundos de vida, de estos discursos institucionales a los que han estado expuestos los sujetos. Sin embargo, cuando se enfrentan registros de expertos y legos, se muestran distantes en sus expresiones sobre el cambio climático (Jasanoff, 2011; Sarabia y Bianchi, 2019). Estos últimos estudios tienen especial relevancia por la distancia que se establece entre el discurso societario (de los medios de comunicación, en particular; de las instituciones, en general) y el discurso social, el que los sujetos expresan en sus nichos comunicacionales cercanos. Este es el marco en el que se instala este trabajo, es decir: a) en la distancia entre discurso societario y discurso social; b) remarcando la reflexión metodológica, sobre cómo unas prácticas de investigación social recogen en mayor medida el discurso societario (análisis de documentos y medios de comunicación, encuestas con cuestionario estandarizado); mientras que otras (entrevistas cualitativas, prácticas grupales) están más abiertas a la recepción de los discursos sociales; c) planteando que la existencia de un discurso societario - está claro que existen instituciones que generan discursos sobre el cambio climático- puede encontrarse con la ausencia de un discurso social sobre el fenómeno; d) y, por último, reflexionando sobre las explicaciones de tal ausencia, especialmente gracias a las aportaciones de la obra de Schutz y su concepto de relevancia.

3. Sobre las «políticas demoscópicas», como concreción de un discurso institucional sobre el cambio climático, véase Baigorri y Caballero (2018). 
En estudios realizados mediante encuesta con cuestionario estandarizado, se muestra una notable preocupación por el fenómeno. Mientras que en aquellos que utilizan métodos cualitativos se destaca la poca implicación de la población en el cambio climático, o incluso se habla de escepticismo cuando los informantes son expuestos a los acuerdos de las grandes conferencias políticas sobre el tema (Wibeck et al., 2017) o ante potenciales medidas a tomar para afrontar el cambio climático (Macnaghten y Szerszynski, 2013; Corner et al., 2013). Cuando las prácticas de observación son suficientemente abiertas, lo que se recogen son dudas, más que certezas (Anshelms y Hulman, 2015). Tal situación aplicada al cambio climático podría ir más allá de este fenómeno específico, y estar concretada en otros fenómenos en los que un discurso societario, institucional, carece de eco en un discurso social.

\section{Contextos concretos de observación}

Los estudios de opinión sobre el cambio climático recogen preocupación por el fenómeno. Para sumergirnos en la relación de la sociedad con esta materia, fijémonos mínimamente en algunos resultados producidos por importantes centros demoscópicos. En el estudio de la primavera de 2015 del Pew Research Center, se observa que la preocupación es muy distinta según la zona del mundo en la que residan los entrevistados, desde el $74 \%$ de Latinoamérica, que opina que el cambio climático es un problema serio, al $18 \%$ que tiene la misma opinión en China, pasando por el $54 \%$ de Europa. Diferencias que parecen recoger la distinta experiencia vital respecto al cambio climático.

En la octava ronda, de 2016, de la Encuesta Social Europea, el porcentaje de europeos muy o extremadamente preocupados es del 25,8\% (21,7\% + 4,7\%). En el caso de los españoles, es del 49,6\%. Parece que existe cierta preocupación por el fenómeno entre la población europea. Ahora bien, cuando se expone el cambio climático entre otros problemas que ha de afrontar la Unión Europea, su posición no está entre las primeras, como muestra el Eurobarómetro de otoño de 2017. Entonces, el cambio climático queda por debajo de la inmigración, el terrorismo, la situación económica, las finanzas de los estados miembros de la UE o el desempleo, entre los problemas que tiene que afrontar la UE. Cuando se pide a los españoles que directamente muestren el grado de preocupación por el asunto, somos los más preocupados. ${ }^{4}$

Estos resultados sirven para enmarcar nuestro objeto: se muestra preocupación por el cambio climático, pero parece tratarse de una preocupación que fácilmente pasa a un segundo nivel cuando se enfrenta el conjunto de problemas de la opinión pública. Como se pone de manifiesto en el gráfico 1 -que representa la evolución desde 1985 de la señalización de los problemas medioambientales entre los principales que existen en España, según los barómetros del Centro de Investigaciones Sociológicas (CIS)_, se observa que,

4. Eurobarómetro Especial 459 sobre Cambio Climático (trabajo de campo, marzo de 2017). 
Gráfico 1. Evolución (1985-2019) porcentaje población española mayor de 18 años que señala como problema: paro, corrupción y fraude y problemas medioambientales

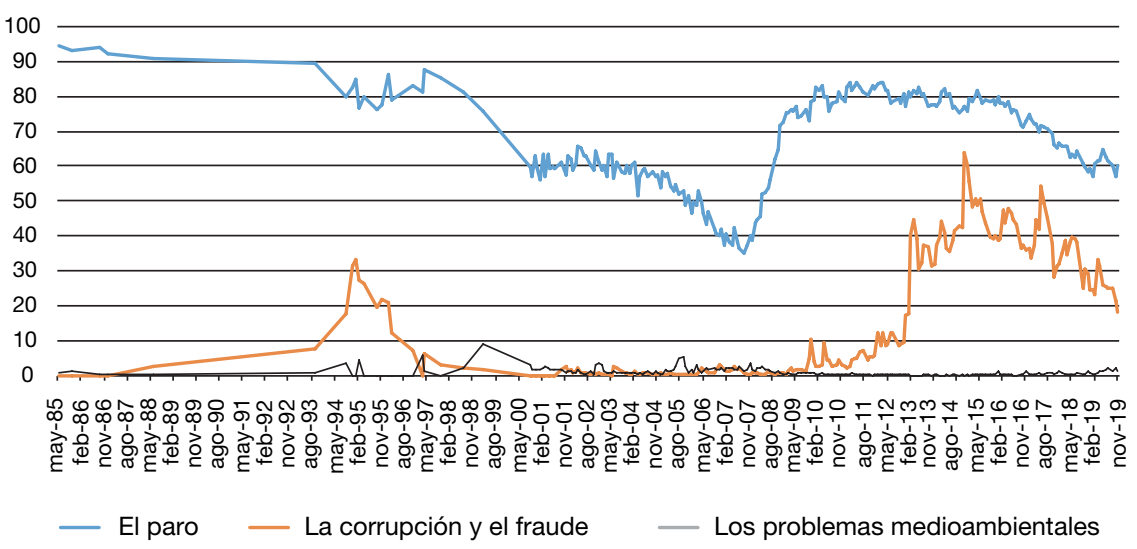

Fuente: elaboración propia a partir de los barómetros mensuales del Centro de Investigaciones.

salvo acontecimientos (inundaciones, accidentes nucleares o medioambientales, sequía), este tipo de problemas ha ocupado un (lejano) segundo orden. Esta representación destaca especialmente cuando se compara con la evolución de problemas como el paro o la corrupción y el fraude.

Siguiendo con los resultados de las encuestas con cuestionario estandarizado del CIS, la ambivalencia de la sociedad, respecto al cambio climático en particular y los problemas medioambientales en general, se muestra de varias formas y a lo largo del tiempo, ya sea en momentos atravesados por la crisis económica de 2009, anteriores o posteriores. Así, se tiene que en enero de 2005 (estudio 2.590) el grado de preocupación por los problemas del medio ambiente es del 6,84, en una escala entre 0 y 10. En abril de 2009 (estudio 2.807), el $85,5 \%$ responde que el cambio climático es algo personalmente bastante o muy importante. En semejante sentido se puede considerar el grado de preocupación $(3,79$, en una escala entre 1 y 5$)$ mostrado por la sociedad española en mayo de 2010 (estudio 2.837), en plena crisis económica. Ya en enero de 2020 (estudio 3.271) y tras la experiencia de la organización de la COP25, el 67,3\% dice seguir con interés las noticias sobre el cambio climático. Sin embargo, y a pesar de que no sean opiniones directamente relacionadas con el cambio climático, hay que apuntar que, en septiembre de 2012, el 15,5\% decía sentir interés y preocupación por los problemas del medio ambiente; el $48,6 \%$, que, aunque esté interesado, no está suficientemente preocupado por tales problemas; mientras que el $32 \%$ manifestaba tener muy poco interés y preocupación. En noviembre de 2016 (estudio 3.159), tales porcentajes eran respectivamente del $14,5 \%$, el $46,3 \%$ y el $35 \%$. Al menos, hay patrones de respuesta, ya sea en plena crisis económica (2012) o cuando ya parecía que se salía de la misma (2016). 
Esta rápida panorámica de resultados de encuestas con cuestionario estandarizado nos muestra una extendida preocupación, pero relativa. Algo que constata la observación empírica realizada, con las siguientes coordenadas: ${ }^{5}$

a) Nueve grupos focalizados, con entre ocho y nueve participantes cada grupo, con un guion con una parte muy abierta al principio, tras plantear cómo veían su futuro en los próximos años. Los perfiles de los grupos realizados entre octubre y diciembre de 2013 fueron los siguientes, de manera que el diseño estuvo estructurado sobre el eje mayor/menor riesgo, que, a su vez, se establecía sobre tres criterios principales, como es el tamaño de la ciudad, el grado de industrialización de la ciudad y el grado de estabilidad laboral. En segundo lugar y superpuestas a las anteriores, quedaban la edad y la tenencia de hijos:

- Funcionarios capital provincia sin riesgos tecnológicos. Hombres. 50-60 años. Valladolid.

- Técnicos y profesionales, en empresas medias. Mujeres. 35-45. Toledo.

- Estudiantes universitarios, clase media-alta. Mixto. 20-25. Valladolid.

- Amas de casa, hábitat macrourbano, clase media, con hijos menores. 35-45 años. Madrid.

- Trabajadoras (dependientas, peluqueras, etcétera), hábitat macrourbano, clases populares, con hijos menores. 30-40 años. Madrid.

- Trabajadores autónomos (sector oficios: fontanero, taxista, dueño de pequeño bar, etcétera), entorno urbano. Varones. 40-50 años. Madrid.

- Empleados contrato fijo (empresa grande o media). Proximidad riesgo tecnoindustrial. Hombres y mujeres. 30-40 años. Tarragona.

- Trabajadores sector industrial-químico. Varones. $45-55$ años. Madrid.

- Jóvenes empleados contrato temporal, sin hijos. Mixto. 20-30 años. Madrid.

b) Encuesta con cuestionario estandarizado, con aplicación telefónica, a una población española mayor de 18 años. El tamaño de la muestra es de 2.400 individuos. En la selección, se aplican cuotas de edad, sexo, región, tamaño del hábitat y ocupación ( $44 \%$ ). Se utilizó un cuestionario con 41 preguntas, con una duración media de 25 minutos y 37 segundos. El trabajo de campo tuvo lugar en mayo de 2014.

c) Cuatro grupos interpelativos, ${ }^{6}$ realizados en Madrid en abril de 2019 y caracterizados con una mayor directividad por parte del moderador y por contar con cuatro participantes. Son cuestionados más directamente sobre sus manifestaciones $\mathrm{y}$, sobre todo, sus representaciones y posiciones con respecto al cambio climático. ${ }^{7} \mathrm{~A}$ diferencia de los grupos focalizados, la

5. Investigación realizada dentro del proyecto Riesgo, incertidumbre y vulnerabilidad en España, financiado por el Plan Nacional (CSO 2010 - 20235). Equipo formado por Ramón Ramos Torre (IP) y Javier Callejo.

6. Investigación realizada dentro del proyecto Incertidumbre y cambio climático (CSO201784007-R).

7. La interpelación se concreta en un cuestionamiento directo de la posición de los interlocutores, un «¿̨ tú....?». 
dinámica estuvo más centrada en las implicaciones de los sujetos en el cambio climático. Los perfiles fueron los siguientes:

- 2 varones ocupados / 2 mujeres ocupadas. Clases medias / medias. Residen en el área metropolitana de Madrid (fuera del municipio de Madrid). Clases medias funcionariales. Dos acuden a su trabajo con transporte privado; dos acuden en transporte público. Con hijos entre 8 y 15 años. 40 y 50 años.

- 2 varones estudiantes universitarios / 2 mujeres estudiantes universitarias. Cursan en facultades distintas materias no relacionas directamente con el cambio climático. Dos vegetarianos. Clases medias. Residen en Madrid (ciudad).

- 2 varones / 2 mujeres. Empleados medios de grandes o medianas empresas (excluidas empresas energéticas, químicas o semejantes) o funcionarios medios de la Administración pública. Clases medias funcionariales. Llevan dos meses buscando casa para cambiar de vivienda habitual. Dos residen en Madrid municipio / 2 en el área metropolitana. 30 y 40 años.

- Mujeres de clases populares en situación de paro. Dos mujeres con hijo/a en la enseñanza secundaria / 2 sin hijos. 35 y 45 años.

\section{Resultados}

Los resultados de la encuesta con cuestionario estandarizado son acordes con los ofrecidos por otras fuentes que utilizan el mismo instrumento, es decir, preguntas administradas mediante cuestionario estandarizado que interrogan directamente sobre el grado de preocupación respecto a un problema públicamente reconocido. El grado de preocupación de la muestra encuestada sobre el calentamiento global es 7,87 (entre 0 y 10). Parece mucho, pero se encuentra por debajo de la preocupación por la pobreza $(8,88)$, el trabajo $(8,83)$, el futuro de las pensiones $(8,32)$, la contaminación del aire o del agua $(8,14)$, la delincuencia $(7,98)$, los residuos tóxicos $(7,89) .{ }^{8}$ Para el $12,2 \%$, el grado de preocupación no supera el 5 (entre 0 y 10 ). ${ }^{9}$

No sería la primera vez que se generan dudas sobre la capacidad de la encuesta para captar algunos de los aspectos de la relación de la sociedad con el medio ambiente (Nisbet, 2011; Wynne, 1996). Sin entrar en el debate sobre la adecuación de este instrumento, lo inicialmente reseñable es la constatación

8. El máximo de NS/NC es de 3 individuos en «el aumento de la población mundial».

9. Ante la muestra de tal grado de preocupación, los que quedan marcados son los que exhiben poca preocupación, el 12,2\% $(\mathrm{N}=293)$ de quienes puntúan su preocupación por el cambio climático por debajo de 6. Realizado un análisis de regresión logística, en el que la variable dependiente es tal preocupación por el cambio climático por debajo de 6 (tabla 6), el perfil de estos despreocupados es: a) aumenta la probabilidad de estar entre los mismos con la edad; b) derecha en el autoposicionamiento ideológico; c) ingresos en el hogar por encima de 3.000 euros al mes; d) ausencia de hijos; e) varones (de hecho, la probabilidad de estar entre los despreocupados es de más del doble para los hombres que para las mujeres). 
de la aparente despreocupación desde la perspectiva cualitativa y la elevada preocupación cuantitativa. Un resultado que ya habría sido observado por estudios anteriores sobre el cambio climático. ${ }^{10}$

Teniendo en cuenta tales resultados, la preocupación de los españoles por el cambio climático es alta. Cabría esperar lo mismo de la perspectiva cualitativa. Pues bien, no aparece mención al fenómeno, ni a asuntos que puedan considerarse temáticamente adyacentes (medio ambiente, contaminación, efecto invernadero, etcétera), en una fase de la dinámica grupal de baja directividad que se alarga más allá de la hora de duración. Además, es relegado a los últimos lugares cuando, ya en una fase directiva de la dinámica, se pedía a los participantes en los grupos que opinaran sobre distintos problemas. Lo que aquí nos interesa realzar es que, en un contexto situacional favorable para hablar del cambio climático, el tema no aparece. Por lo tanto, se puede leer como síntoma de la relación de la sociedad con este fenómeno y parece señalar directamente a la debilidad de la preocupación.

Entre las estrategias de combinación de métodos ${ }^{11}$ —aquellas que combinan las aproximaciones cualitativa y cuantitativa de la investigación social—, llama la atención la connotación negativa de los términos utilizados para denominar este acontecimiento empírico: divergencia, inconsistencia, disonancia (Rossman y Wilson, 1985). En la primera oleada ${ }^{12}$ de trabajos de reflexión metodológica sobre esta estrategia, en los años noventa del siglo pasado, el acontecimiento se explica como un problema que está en los investigadores y los recursos utilizados (Cresswell y Plano, 2011; Bericat, 1998). Es algo que hace demasiado ruido, puesto que lo que se busca en las estrategias de combinación de métodos ${ }^{13}$ es la integración de datos. Como el objetivo es legitimar la propuesta de los diseños de estrategia combinada, se da prioridad a la convergencia, a lo que ofrece la propia combinación. Incluso hay autores que hablan de correlación (Onwuegbuzie y Teddlie, 2003: 366). ${ }^{14}$ El espíritu de la estrategia de combinación de métodos se dirige hacia la convergencia, y la divergencia hace ruido. El consejo es más descriptivo que una base para la explicación: exponer las convergencias y las divergencias (Lee y Greene, 2007; Slomi-Nevo y Nevo,

10. Norgaard (2011b: 182) constata la distancia entre los informes de resultados de encuestas de opinión y entrevistas en profundidad o focus group. Stoll-Keeman et al. (2001) utilizan grupos focalizados para abordar la inacción en Suiza, a pesar de los resultados de las encuestas de opinión.

11. En inglés, se ha impuesto el término mixed methods.

12. En el manual de referencia de las estrategias de combinación de métodos (Tashakkori y Teddlie, 2003), ya aparecen evidentes huellas del problema y se formula una curiosa solución: llevar a cabo nuevos estudios en que se reformulen las preguntas (Onwuegbuzie y Teddlie, 2003: 378).

13. Siguiendo un texto ya clásico en este tema (Tashakkori y Teddlie, 1998), se opta por la denominación combinación como término general para referirse al uso conjunto de métodos cuantitativos y cualitativos.

14. De manera crítica, Witcher et al. (2001). 
2009). ${ }^{15}$ Un paso más dan Cresswell y Plano (2011), pues, en caso de divergencia, recomiendan dar prioridad a los temas en los que hay más convergencia que divergencia.

En todo el recorrido de más de un cuarto de siglo de reflexión metodológica sobre las estrategias de combinación de métodos, si no se ajustan los resultados es porque hay un error en el investigador (Rossman y Wilson, 1985: 633; Erzberger y Kelle, 2003: 475). No obstante, en los últimos autores pasa de considerarse la divergencia como un indicador exclusivamente resultante de la pobreza del diseño de estudio a una potencial fuente para la producción teórica en este campo. Parece muy pertinente el consejo de Bryman (1988) —uno de los primeros sociólogos que se enfrentan a la posibilidad de divergencia cuando se utilizan métodos cualitativos y cuantitativos en una misma investigación-, que reza: habrá que buscar nuevas formas de preguntar.

Tras la ausencia de referencia espontánea al cambio climático en los grupos de discusión, se cuestiona a los participantes por el fenómeno social. En la respuesta, se erigen dos categorías de justificaciones, muy entrelazadas. Un primer momento, producto de la reacción a la interpelación, en el que se diferencian tres tipos de respuesta, que suponen tres rasgos de la representación que los participantes en los grupos tienen del cambio climático:

a) Como realidad débil: «El cambio climático es una realidad, o sea, no se puede decir que no, lo que yo no tengo claro es que sea tan negativo» (funcionarios). Justificación que no problematiza lo dicho por el sistema experto, al contrario de lo expuesto por Wynne (1996: 50), cuando estudia conflictos medioambientales concretos. Según declaraciones como la recogida, se muestra cierta confianza reflexiva en el mismo (Giddens, 2008), en una especie de manifestación que dialoga con las negaciones de esa realidad, que, a su vez, cuestionan la línea dominante del sistema experto en este tema. La expresión «no se puede decir que no» sitúa a los que niegan el cambio climático en la respuesta. Ahora bien, también se atenúa la importancia de los daños: «Lo que ya no tengo tan claro es que sea tan negativo".

b) Una realidad que preocupa... poco, como una preocupación secundaria: «No es que no nos preocupe, lo pensamos de vez en cuando, pero es que con esta situación» (trabajadoras clases populares).

c) Una realidad no inmediata, distanciada en el tiempo, lo que es una distancia sobre su concreción: «No quiero decir eso, no. Y decir: Y se va a comer

15. Seguramente, por el impulso pragmático que domina esta propuesta metodológica, en la que se intenta reflexionar sobre una variedad de experiencias de investigación que se venían haciendo desde hace mucho, sin que encajaran bajo ninguna categoría clasificatoria en la polaridad cuantitativo-cualitativo, las salidas que se dan a la experiencia de la divergencia son también de corte pragmático. Así, Slomi-Nevo y Nevo (2009) proponen que se dé preferencia al método —entre los dos_ que acierte. La solución es, desde luego, aplastante. El problema es qué significa acertar y cómo se alcanza la certeza de que una observación es acertada. Por supuesto, entonces, se rechazaría la desacertada. 
agua, terreno, y las playas van a desaparecer y tal. Pero una cosa como a muy largo plazo» (técnicas y profesionales).

Representación del cambio climático como realidad latente cuya manifestación se distancia en el tiempo. Después y tras estas justificaciones inmediatas, se registran algunas atravesadas de mayor racionalización, lo que permite considerar que es aquí donde se encuentra la justificación en sentido estricto, pues lo que hace es situar el fenómeno cuestionado en el que es percibido como su justo lugar, detrás de otros problemas. La principal línea ${ }^{16}$ que se traza es la que distribuye lo prioritario - «lo que se te viene encima»de lo que no lo es, donde se encuentra el cambio climático. No es la línea que separa lo importante o lo preocupante de lo que no es ni importante ni preocupante.

Cuando parece que las justificaciones se van a elevar a discurso, se detienen en la línea trazada: se bloquea su posibilidad de discurso y se funden nuevamente con el silencio. Los grupos focalizados no hablan del cambio climático, ni el cambio climático hace hablar a los grupos. Las justificaciones dadas se consideran suficientes. Hay que anotar que en estas justificaciones no hay demanda, no hay lesiones, no hay víctimas, no hay daños, lo que permite, por un lado, clasificarlo como sin prioridad. Por otro lado, y esto es parte de nuestra tesis, impide que el cambio climático genere discurso: «No, pero no te ves afectada directamente. La pobreza te afecta directamente, el paro te afecta directamente» $\mathrm{o}$ "Las otras te afectan individualmente» (técnicas y profesionales).

Estas justificaciones son asumidas grupalmente, después de cuestionar la ausencia de discurso.

Los grupos interpelativos, más centrados en la búsqueda de explicación de la ausencia de referencias al cambio climático entre las preocupaciones, confirman su ausencia como discurso:

— Moderador: ¿̨e habla de esto, normalmente, en una conversación cotidiana?

- Mujer 1: No, no es algo... No es un tema de conversación.

- Hombre 2: Vamos, no está entre los primeros cien temas de conversación... [risas], yo creo que es la primera vez en mi vida que hablé del cambio climático [risas].

- Mujer 1: Tanto rato. Porque sí que puedes decir: «Bah, es una chorrada», «oye, es una chorrada eso» o "se va acabar el mundo, tal». Y ya se acaba la conversación y te vas a otra cosa.

- Hombre 1: Yo lo único que hablo es que digo: «Joder, mira, ayer estábamos con rebeca y hoy estamos en manga corta, a ver el cambio climático». Comentario tonto, absurdo... (Residentes área metropolitana)

16. En el sentido de Zerubavel (1993). 


\section{Hacia la interpretación del no hablar y las condiciones del discurso social}

El silencio sobre el cambio climático no es atribuible a imposiciones manifiestas (Bourdieu, 1985: 51), tabúes o ritos de exclusión (Foucault, 1974: 14; Burke, 1996), ni cabe explicarlo como fruto de la regulación de las interacciones (Wardhaug, 1986; Hymes, 1986; Basso, 1972). Y aun cuando el esfuerzo de información por parte de instituciones y medios de comunicación difícilmente puede calificarse de exitoso, ${ }^{17}$ el silencio recogido no parece estar relacionado con la ignorancia: saben de la existencia de algo que es el cambio climático, aunque tal vez no se desee saber mucho más. Como si lo que supieran fuese considerado suficiente. Así se pone de manifiesto en los estudios recogidos por Gross (2010), Gross y Heindrich (2010) y Gross y McGoes (2015). Se trata de un saber que les sirve para seguir funcionando (Leitner, 2012).

Desde los estudios realizados con metodología cualitativa, el silencio se ha explicado como fruto de un proceso de denegación (Optow y Weiss, 2000; Norgaard, 2001a). No se desea reconocer una responsabilidad-culpa, ya que hacerlo exige renunciar a posiciones de privilegio o condiciones de bienestar, en definitiva, asumir responsabilidades. Sin embargo, no parece que el silencio registrado en esta investigación derive de una activa evitación (Zerubavel, 2006: 12). ¿Qué tienen que tapar nuestros grupos?

Como se ha señalado en las justificaciones, tampoco se dibujan víctimas, ni referencias a algo que pueda ser entendido como daño causado. La víctima es un concepto sin sujeto, es el planeta, que, como muestra la siguiente frase que utiliza la forma reflexiva, parece estar destruyéndose a sí mismo: «Mientras no haya una reacción, yo creo que nosotros no nos vamos a dar cuenta de que el planeta se está destruyendo» (grupo interpelativo empleados).

Se erige así la tesis de la inexistencia de un discurso en la sociedad —entre los legos o no expertos- sobre el cambio climático. Y esta tesis se sostiene a partir de dos asunciones que se encuentran entrelazadas: no hay discurso porque no hay demanda de acción sobre este fenómeno, y no hay discurso porque no se perciben sujetos presentes en el cambio climático.

En toda demanda social, se encuentra lo que Boltanski (2014: 180) denomina gramática de la acusación, resultante de una experiencia vivida o representada como injusta. En las justificaciones, esto no se registra. Los interpelados, por supuesto, no se sienten víctimas de una injusticia,

17. Con respecto al «ineficaz» esfuerzo de la comunicación científica en general por hacer llegar sus mensajes al conjunto de la población, véase Sloman y Fernbach (2017: 184 y ss.). ¿Cómo se explica la ignorancia sobre el cambio climático, a pesar de toda la información? Es algo que ahora (Corner, 2018) se pregunta el experto IPCC (Panel Internacional sobre el Cambio Climático). Al respecto, Uekotter y Lübken (2014) subrayan que la ignorancia se mantiene a pesar de una aparatosa gran presencia de información y conocimiento, con una alusión a la tesis de Luhmann (1989; 1997) de la fragmentación del conocimiento, de manera que hay mucho conocimiento, pero fragmentado en especializaciones, y mucha ignorancia en común, incluso entre expertos. 
pero tampoco señalan a otros como víctimas. No hay demanda porque no existe la concepción del cambio climático como generador de daños, lesiones, desigualdad e injusticia, como también señala el estudio de Polski y Eakin (2011).

La otra causa de la inexistencia del discurso tiene que ver con el sujeto. No puede decirse que los observados se sientan sujetos con relación al cambio climático. Las huellas sostenidas en las transcripciones muestran, al contrario, que no se representan como víctimas. Pero tampoco como causantes, a pesar de las leves discusiones producidas sobre el carácter antropogénico del cambio climático. ${ }^{18} \mathrm{La}$ ausencia de sujeto es explicada (Szerszynski et al., 1996; Jasanoff, 2010) como consecuencia del hincapié en soluciones tecnológicas, que expulsa la acción humana de la solución. En la observación empírica realizada, no hay sujetos como víctimas o causantes, ni tampoco como mediadores. En las justificaciones recogidas, apenas existe una nebulosa referencia al «gestor».

Ante las evidencias recogidas, cabe preguntarse qué es un discurso para la sociología y qué hace que un fenómeno social se convierta o no en discurso. Buena parte de los ejercicios de presentación del análisis del discurso (véase, sobre todo, Dijk, 1990), reforzando su sentido pragmático, consisten en aplicaciones del mismo en variados contextos y soportes (las instituciones, el poder, la novela, la conversación, los medios de comunicación, las organizaciones, etcétera), con la finalidad de desvelar el uso del lenguaje como instrumento de dominación, cuando se trata del análisis crítico del discurso (ACD). Sin menoscabo alguno de la notable contribución, las actitudes y el horizonte que se encuentran tras la gran mayoría de estos trabajos, también recogen materiales que definen como discurso para integrarlos en lo que sí es un discurso, presente en numerosas y amplias líneas de investigación lingüística, que demandan a sus ejercientes que desvelen cómo se implementa la dominación a través del lenguaje, donde el concepto de discurso es asumido, pero no definido, más allá de como concreción lingüística. ${ }^{19}$

18. Esta baja implicación tal vez explique que los españoles se encuentran entre los ciudadanos europeos que en menor medida dicen llevar a cabo comportamientos relacionados con el reciclaje de residuos: 62,6\% (Meira, 2009: 94); 73,5\% (Departamento de Medio Ambiente del Gobierno de Navarra 2000, solo sobre población de Navarra); 76,8 \% (Ecobarómetro Andalucía 2008, solo población de Andalucía); 31,6\% (DXDS 2003, solo población de Galicia); 49 \% (Eurobarómetro Social 2001, solo población del País Vasco). El porcentaje de los que reciclan (lo hacen siempre o bastante), según el estudio del BBVA de 2013, es del $71 \%$ — por debajo de Alemania (91\%), Francia (90\%), Italia (89\%), los Países Bajos (91\%), Suecia (90\%), Dinamarca (85\%) y el Reino Unido (87\%).

19. Las revistas sociolingüísticas hacen más hincapié en la metodología o en los canales del discurso (oral, medios de comunicación, políticos en sede parlamentaria, etcétera) que en la exposición de una definición de discurso, de manera que toda expresión lingüística —y no lingüística - es susceptible de ser asumida como discurso. Así, el término discurso ha pasado de ser una de las posibles concreciones de la lengua a la concreción de las lenguas. Entre las revistas en este campo, véase Discourse Studies, Discourse \& Communication, Language and Speech, Communication and the Public. 
No todo lo que entra en la conversación o el texto puede considerarse discurso. ${ }^{20}$ Debido al ascendente de Foucault sobre las disciplinas sociales en general y sobre las lingüísticas en particular, que abrió ampliamente la puerta de las mismas hacia una concepción política y social de las prácticas expresivas, se ha extendido - creo que en exceso- el uso del término discurso, hasta el punto de hacer de cualquier habla un discurso. Y este exceso está en el propio Foucault. ${ }^{21}$ Parece más ajustada la distinción que establece Zizek (2001: 66) entre "habla en sí y habla para sí», en la que el discurso puede entenderse como «habla para sí». La distinción permite diferenciar entre discurso social, que sitúa al hablante como sujeto frente a un objeto tematizado, de expresiones que en nada o casi nada implican al sujeto con respecto al objeto cuestionado. Esto nos permite conectar con lo que Boltanski denomina gramática de la acusación (demanda, según los términos utilizados aquí), en oposición a una gramática de la justificación ${ }^{22}$ (defensa de la demanda, respuesta a la demanda, contrademanda). ${ }^{23}$ Un discurso social es un discurso "para sín, que sitúa al hablante en una gramática de la acusación o de la justificación.

La gramática de la acusación ha de venir a atenuar la tensión existente entre la puesta en práctica de una violencia y la correspondiente petición de justicia,

20. Así, se habla del discurso de la cortesía para hablar de la gramática de las formas corteses, es decir, de cómo el habla genera igualdad o desigualdades o las interrogaciones policiales. El habla como instrumento social, con sus implícitos de exclusión o dominación. No niego eso, pero creo que para llamarlo discurso tiene que haber algo más. Es más, tales consideraciones de las hablas como instrumento de dominación solo pueden entenderse desde los discursos que enmarcan tales análisis y situaciones.

21. En sus estudios genealógicos sobre la constitución de la sexualidad en objeto disciplinar (Foucault, 1980: 29), señala el imperativo de convertir el sexo en habla, especialmente por parte de la Iglesia, al obligar a poner el sexo en la palabra, pero hacerlo bajo una disciplina. Ahora bien, el sexo en la palabra no es el discurso. El discurso es el que establece la imposición (demanda un hacer) al conjunto de los ciudadanos (sujetos) en función de argumentos. El habla del sexo se inscribe en un discurso del sexo, pero el habla de los sujetos no es el discurso, sino, a lo sumo, la concreción de un discurso ajeno (como diría Bajtin, en Voloshinov, 1993). El reto está en distinguir entre habla, discurso y concreción de los discursos en el habla, de manera que se defina el estatuto del último. Foucault (1984) es consciente de la extensión que hace del concepto de discurso cuando critica el limitado uso que hacen de él los lingüistas pragmatistas.

22. Casi todo discurso de contrademanda, de contestación a una demanda, se erige en justificación.

23. Entre las variantes acciones de defensa de los demandados, en la resistencia a admitir su culpabilidad, están: $a$ ) admitir la legitimidad de los valores que sostienen la acusación y la realización de los hechos exponiendo excusas, en lugar de justificaciones o legitimidades que se opongan a la argumentada por la acusación ("lo hice sin querer, debido a determinadas circunstancias»); b) admitir la legitimidad de los valores que sostienen la acusación, pero negar la acción imputada ("nunca me he comportado como un machista»); $c$ ) admitir la legitimidad de los valores que sostienen la acusación, negar como propias las acciones imputadas, pero comprender a quienes llevan a cabo tales acciones, con lo que el hablante abre la sospecha sobre la legitimidad inicialmente admitida («yo no soy racista, pero...»); d) negar la legitimidad de los valores que la sostienen («las parejas homosexuales no pueden tener los mismos derechos», luego no han de tener ningún derecho). 
la gramática de la justificación tratará de compatibilizar las exigencias de común humanidad de los actores (que presupone su igualdad) y la ordenación jerárquica de los mismos. (Boltanski, 2014: 180)

La demanda surge de un padecimiento, de una injusticia, en términos de Boltanski (2014). Se propone que el discurso social requiere de unas exigencias semejantes a las presentes en la acción de las instituciones jurídicas. ${ }^{24}$ Para la sociología, el discurso social, presente en los nichos de comunicación de los sujetos, conlleva un juicio. No solo entonado como opinión o valoración sobre algo, sino como demanda de justicia, como un juicio inserto en un litigio, en una disputa, en un conflicto, ${ }^{25}$ y por lo tanto como un discurso en el que también está inserto — e implicado- el sujeto, puesto que es un «discurso-parasí». Tal lógica judicial del discurso social sirve al análisis sociológico empírico para detectar conflictos, en que una de las partes, al menos, se percibe como sujeto de derechos conculcados, ${ }^{26}$ y a otras partes - mediadores, instituciones, actores políticos — se les demanda detener o restituir la acción apropiadora de derechos. Desde esta perspectiva, los discursos sociales reclaman acción o contra-acción (parar la acción reclamada por otros), por lo que reafirman su carácter estratégico (Foucault, 1984b).

¿Se puede admitir como "discurso sociológicamente relevante» una frase, tomada de Ricoeur, como: "Una baja presión atmosférica en tal punto del globo es responsable de una tempestad...» (Ricoeur, 1988: 68). Hay sujetos, pero no hay demanda. ${ }^{27}$ En cuanto a demanda frente a otro y dadas sus características judiciales, todo discurso viene y lleva al enfrentamiento, y no al consenso. El sujeto se constituye en relación con otros sujetos o como respuesta a otros que lo han preconstituido como sujeto, como interpelado. Un sujeto que se hace soporte/portavoz de un valor, de un grandeur (Boltanski y Thévenot, 1991). El discurso social se establece así sobre una demanda y unos sujetos; los sujetos porque mantienen discursos que demandan, y las demandas sociales se expresan conflictivamente en discursos que sitúan sujetos, de manera que estos devienen coherentes (Alonso, 1998: 223).

El tramo recorrido en el intento por fijar qué es un discurso social sirve ahora para intentar explicar la relación entre sociedad y un fenómeno, cuando se reconoce que el mismo apenas tiene cabida en esos discursos sociales, en lo que habla la gente en sus nichos comunicativos. Una muy sintética

24. Intuición presente en Ricoeur (1988: 63): «Las transiciones entre las proposiciones del lenguaje ordinario sin un tinte moral o jurídico y las decisiones jurídicas están aseguradas por proposiciones del lenguaje que reivindican, confieren, transfieren, reconocen, atribuyen derechos y que tienen la forma esto es tuyo, vuestro, mio».

25. El derecho es una ritualización de la guerra (Foucault, 1984: 69).

26. Aun cuando se ha venido utilizando aquí el término víctima, con mayor eco y, por lo tanto, capacidad reflexiva en el conjunto de la sociedad, tiene mayor pertinencia el concepto «objeto de adscripción de derechos» (Hart, 1992: nota 23). Por otro lado, si se trata de los propios hablantes y más allá de la pertinencia de la categorización jurídica, son pacientes que reclaman devolución de derechos.

27. Salvo que se entienda como demanda la solicitud de la intervención divina. 
aproximación a las distintas explicaciones sociológicas sobre el silencio puede ayudar:

- El silencio impuesto

- El silencio por irrelevancia

— El silencio latente por sedimentación del fenómeno

El silencio impuesto por conflicto latente es seguramente el que antes acude a nuestro pensamiento cuando se señala algo como callado. Es el producto de la censura (Bourdieu, 1985: 51), ya sea fruto de la explícita prohibición, o de la implícita conjura en la denegación - como se observa en Norgaard (2011a) y Zerubavel (2006) — o ya sea porque no es socialmente aceptable, pues se puede encender el conflicto entre los hablantes.

En el silencio por irrelevancia del tema, lo irrelevante es, a la vez, existente y reconocible, pero carente de capacidad suficiente para captar el trabajo de la conciencia del sujeto (Schutz, 1996). Situado fuera de la conciencia, sin interés, se ubica también fuera del habla y, más aún, de un «habla para sí». Con la ayuda de Luckman, Schutz (Schutz y Luckman, 1973) ${ }^{28}$ dibuja las características de la irrelevancia, de las que se destacan las que son de especial interés aquí:

- Lo que está fuera de nuestro alcance (Schutz y Luckman, 1973: 54).

- Sobre lo que no se discute. Lo no problemático, frente a lo significativo (Schutz y Luckmann, 1973: 182).

El concepto de relevancia tópica de Schutz (1970: 26) nos sirve para abordar el silencio latente: algo que, de lo aproblemático familiar, ${ }^{29}$ aparece como problema y se convierte en tema. Algo incuestionado pasa a ser cuestionado. Se segrega de lo incuestionable y se impone como relevancia impuesta ${ }^{30}$ (Schutz, 1970: 28). Pasada la fase de relevancia impuesta, vuelve a lo familiar y se considera ya resuelto. Este es un proceso que Schutz denomina sedimentación. Silenciado, lo relevante queda sedimentado. Sedimentado, lo relevante queda en silencio, sin especial interés para los sujetos $\mathrm{y}$, en su proyección al conjunto de la sociedad, sin interés para los ciudadanos.

A partir del proceso de sedimentación, un tema antes relevante puede desaparecer como tal porque: a) no es percibido como un problema; b) ha dejado de existir. Por otro lado, ese proceso de sedimentación puede ser interrumpido (Schutz, 1970: 114 y ss.). Cuando el tema relevante está sedimentado produce silencio, pero eso no quiere decir que no sea relevante.

28. Aun cuando puede considerarse que no queda excesivamente lejos de sociolingüistas como Grice (1989) y Sperber y Wilson (1994), focalizados en una economía significativa de los mensajes, la distinción relevante/irrelevante se establece a partir de la fenomenología de Schutz. Lo no relevante se desarrolla en Schutz y Luckman (1973: 187 y ss.).

29. Familiar: un elemento o tema en la actualidad en el mundo incuestionado, pero que puede ser cuestionable (Schutz, 1970: 65).

30. Schutz (1970) diferencia tres tipos de relevancia: impuesta, interpretativa y motivacional. 
De la propuesta de Schutz sobre la relevancia, que califica como el concepto central de la sociología, interesa el proceso de cómo lo relevante se transforma desde el fondo, de lo inapercibido. Una relevancia que se experimenta como realidad social, como "naturaleza», como lo pre-dado (pre-given). A partir de estas consideraciones, el silencio sobre el cambio climático, registrado en la observación empírica, cobra sentido como silencio vacío. Se trata de un silencio sobre un fenómeno que se concibe relevante por lo siguiente: la propia declaración de importancia en las encuestas y en la justificación de los grupos; la asunción de la obligación de responder cuando se inquiere por el tema, y los picos - motivados por acontecimientos medioambientales, que hacen salir al problema de lo totalmente sedimentado- ofrecidos en el gráfico 1.

\section{Conclusiones tentativas}

En los momentos del trabajo de campo, no hay un discurso social sobre el cambio climático en la sociedad española. A pesar de que distintos autores (Douglas y Wildawski, 1982; Szerszynski, 1996) han situado la preocupación por la naturaleza en el centro de nuestras sociedades, no puede decirse que tal preocupación se haya traducido en discurso. La ausencia de un discurso sobre el cambio climático, entre sectores centrales de la sociedad española, puede explicarse por la carencia de sujetos y disputas. Que no haya discurso no quiere decir que no se perciba el fenómeno como un problema relevante, sino que se trata de una relevancia latente, envuelta en una preocupación moderada, postergada entre otras preocupaciones. No está en las prioridades de la ciudadanía. Otra cuestión para indagar en futuras investigaciones son las razones que hacen que los ciudadanos conduzcan los problemas medioambientales en general y el cambio climático en particular -incluso tras poco tiempo después de algún acontecimiento medioambiental con rasgos de catástrofe- desde lo problemático hacia ámbitos de lo poco o menos problemático.

Debido a tal relevancia latente, sedimentada, los detalles de la información recibida son enormemente filtrados en la recepción (Downs, 1972). En la justificación solicitada a los participantes en los grupos, se han mostrado huellas de tal relevancia latente y se ha señalando el notable papel que tiene en la misma la desubicación temporal del fenómeno.

La ausencia de discurso nos lleva a una falta de implicación de la sociedad, que queda reducida a la expresión de preocupación (García, 2004: 283), pero, sobre todo, es un síntoma del grado de incorporación de un fenómeno por parte de la sociedad. A su vez, el silencio gestiona el fenómeno, ya que si no se habla del mismo cabe entenderlo como resultado de su fijación en esa situación de sedimentación. Desde el nivel metodológico, callar permite observar. Las prácticas de investigación social que tienden al silencio del observador pueden recoger el silencio de los observados, de la sociedad observada, que no tiene discurso sobre el cambio climático, con lo que ello tiene de base para inferir su grado de implicación práctica con este fenómeno. 
Desde el diseño metodológico, la producción de silencio a partir de unos métodos de investigación social nos permite enmarcar lo que se dice y recoge desde los otros métodos de investigación. ${ }^{31} \mathrm{El}$ moralizado marco (Eder, 1996: 209) mediático en el que aparece la preocupación sobre el cambio climático (Bell, 1994; Ungar, 1992; Grundmann, 2006; 2007) hace que los respondientes muestren preocupación e impide la no respuesta en la inquisición directa mediante cuestionario, aunque el fenómeno esté envuelto en silencio en sus mundos de vida.

\section{Agradecimientos}

Quiero agradecer aquí la colaboración recibida de todos los miembros del equipo del proyecto Incertidumbre y cambio climático (CSO2017-84007-R). En especial, al profesor Ramos, por su liderazgo en la reflexión sobre las incertidumbres en nuestras sociedad, y a los profesores Agudo, Luque y Rodríguez Victoriano, pues fue entre los cuatro que armamos la propuesta metodológica de los grupos que hemos denominado grupos interpelativos.

\section{Financiación}

Este trabajo forma parte de la investigación realizada dentro del proyecto Incertidumbre y cambio climático (CSO2017-84007-R). Investigación financiada por el Ministerio de Ciencia, Innovación y Universidades. Equipo formado por Javier Callejo (IP), Yolanda Agudo (UNED), Pablo Francescutti (URJC), Ernest García (UV), Emilio Luque (UNED), Ramón Ramos (UCM), Marina Requena (UV) y José Manuel Rodríguez (UV).

\section{Referencias bibliográficas}

Alonso, Luis Enrique (1998). La mirada cualitativa. Madrid: Fundamentos.

Anshelms, Jonas y Hulman, Martin (2015). Discourses of Global Climate Change. Cambridge: Polity Press.

Antilla, Liisa (2005). "Climate of scepticism: US newspaper coverage of the science of climate change». Global Environmental Change, 15 (4), 338-352. <https://doi.org/10.1016/j.gloenvcha.2005.08.003>

Baigorri, Artemio y Caballero, Manuela (20I8). «Negacionismo, políticas demoscópicas y currículum de fracasos. El caso del cambio climático en España». Aposta, 77, 8-58.

<http://apostadigital.com/revistav3/hemeroteca/mcg2.pdf>

Basso, Keith H. (1972). «To give up on words: Silence in Western Apache culture». En: Giglioli, P. P. (ed.). Language and social context. Harmondsworth, UK: Penguin Books, 67-86.

31. Freud (principalmente 1974, 1985), cuya obra versa fundamentalmente sobre técnicas, llegó al psicoanálisis tras el fracaso de probar con la dominación hipnótica de Charcot. Se llega al potencial del silencio de las prácticas cualitativas de investigación social a partir de las dudas en el uso de las encuestas con cuestionario estandarizado, que actúan como la dominación hipnótica. 
Bell, Alan (1994). "Climate of opinion. Public and media discourse on the global environment». Discourse and Society, 5 (1), 33-64. <https://doi.org/10.1177/0957926594005001003>

BERICAT, Edurardo (1998). La integración de los métodos cuantitativo y cualitativo en la investigación social. Barcelona: Ariel.

Boltanski, Luc (2014). De la crítica. Madrid: Akal. <https://doi.org/10.4000/books.res.171>

Boltanski, Luc y Thévenot, Laurent (1991). De la justification. París: Gallimard.

Bostrom, Ann; Morgan, M. Granger; Fischhoff, Baruch y Read, Daniel (1991). "What do People Know about Global Climate Change». Global Environmental Change, 1, 183-208.

Bourdieu, Pierre (1985). ¿Qué significa hablar? Economía de los intercambios lingüisticos. Madrid: Akal.

Boyкоғf, Maxwell T. (2008). «Lost in translation? The United States television news coverage of anthropogenic climate change 1995-2004». Climate Change, 86, 1-11. <https://doi.org/10.1007/s10584-007-9299-3>

- (2013). «Public Enemy No. 1? Understandings Media Representations of Outlier Views of Climate Change». American Behavioral Scientist, 57, 796-817. <https://doi.org/10.1177/0002764213476846>

Boyкоғғ, Maxwell T. y Boyкoff, Jules M. (2004). «Balance as bias: Global warming in the US prestige press». Global Environmental Change, 14, 125-136. <https://doi.org/10.1016/j.gloenvcha.2003.10.001>

Boykoff, Maxwell T. y RoberTs, J. Timmons (2007). «Media Coverage of Climate Change: Current Trends, Strengths, Weaknesses». Human Development Report 2007/2008. <http://hdr.undp.org/sites/default/files/boykoff_maxwell_and_roberts_j._timmons.pdf>.

Brechin, Steven R. (2008). «Ostriches and Changes: A response to Global Warming and Sociology». Current Sociology, 56, 467-474. <https://doi.org/10.1177/0011392107088239>

Bryman, Allan (1988). Quantity and Quality in Social Research. Londres: Unwin Hyman. Burke, Peter (1996). Hablar y callar. Funciones sociales del lenguaje. Barcelona: Gedisa.

Carvalho, Anabela (2005). "Representing the politics of greenhouse effect: discursive strategies in the British media». Critical Discourse Studies, 2 (1), 1-29. <https://doi.org/10.1080/17405900500052143>

- (2010). «Media(ted) discourses and climate change: A focus on political subjectivity and (dis)engagement». Climate Change, 1 (2). $<$ https://doi.org/10.1002/wcc.13>

Corner, Adam (2018). "Communication the science is a step much-needed for UN climate panel». The Guardian, 30 de enero, <https://www.theguardian.com/environment/2018/jan/30/communicating-the-science-is-a-much-needed-step-forun-climate-panel>.

Corner, Adam; Parkhill, Karen; Pidgeon, Nick y Vaughan, Naomi E. (2013). "Meaning with nature? Exploring public perceptions of geoengineering in the UK». Global Environmental Change, 23, 938-47. <https://doi.org/10.1016/j.gloenvcha.2013.06.002>

Cresswell, John W. y Plano Clark, Vicki L. (2011). Designing and conducting mixed methods research. Thousand Oaks: Sage.

Dickens, Peter (1992). Society and Nature: Toward a Green Social Theory. Temple: Temple University Press. 
Dijk, Teun van (1990). La noticia como discurso. Barcelona: Paidós.

Douglas, Mary y Wildawsky, Aaron (1982). Risk and Culture. Berkeley: University of California Press.

Downs, Anthony (1972). «Up and down with ecology-the issue-attention cycle». Public Interest, verano de 1972, 28, 38-50. <https://www.nationalaffairs.com/storage/ app/uploads/public/58e/1a4/b56/58e1a4b56d25f917699992.pdf> [consultado el $15 / 11 / 2019]$.

Dunlap, Riley E. y Brulle, Robert J. (2015). Climate Change and Society: Sociological Perspective. Oxford: Oxford University Press. <https://doi.org/10.1093/acprof:oso/9780199356102.001.0001>

EDER, Klaus (1996). "The institutionalization of environmentalism: ecological discourse and the second transformation of Public Sphere». En: Lash, Scott; SzERszINSKY, Bronislaw y Wynne, Brian (eds.) (1996). Risk, Environment and Modernity: Towards a New Ecology. Londres: Sage, 203-223.

Erzberger, Christian y Kelle, Udo (2003). «Making Inferences in Mixed Methods: The Rules of Integration». En: Tashakrori, Abbas y Teddle, Charles (eds.). Handbook of Mixed Methods in Social \& Behavioral Research. Thousand Oaks: Sage.

Fernández-Reyes, Rogelio; Piñuel-Raigada, José Luis y Vicente-Mariño, Miguel (2015). «La cobertura periodística del cambio climático y del calentamiento global en El País, El Mundo y La Vanguardia». Revista Latina de Comunicación Social, $70,122-140$. <https://doi.org/10.4185/RLCS-2015-1038>

FIll, Alwin y MüHlhäusler, Peter (eds.) (2001). The Ecolinguistics Reader. Language, Ecology and Environment. Londres: Continuum.

Foucault, Michel (1974). El orden del discurso. Barcelona: Tusquets.

- (1980). La voluntad de saber. Madrid: Siglo XXI.

- (1984). La verdad y las formas jurídicas. Barcelona: Gedisa.

FreUd, Sigmund (1974). Esquema del psicoanálisis y otros escritos de doctrina psicoanalítica. Madrid: Alianza.

- (1985). La interpretación de los sueños. Barcelona: Planeta-Agostini (e. o. 1900).

Fundación BBVA (2013). Values and Worldviews: Visión sobre la Naturaleza y el Medio Ambiente. Madrid: Departamento de Estudios y Opinión Pública de la Fundación BBVA.

García, Ernest (2004). Medio ambiente y sociedad: la civilización industrial y los límites del planeta. Madrid: Alianza.

Giddens, Anthony (2008). Consecuencias de la modernidad. Madrid: Alianza.

Greer, Jed y Bruno, Kenny (1996). Greenwash. The Reality behind Corporate Environmentalism. Nueva York y Penang: The Apex Press and Third World Network.

Grice, Paul H. (1989). Studies in the way of the words. Cambridge (Mass.): Harvard University Press.

Gross, Matthias (2010). Ignorance and Surprise. Science, Society and Ecological Design. Cambridge (Mass.): MIT Press. <https://doi.org/10.7551/mitpress/9780262013482.001.0001>

Gross, Matthias y Heindrich, Harald (2010). Environmental Sociology. Dordrecht: Springer. <https://doi.org/10.1007/978-90-481-8730-0>

Gross, Matthias y McGoes, Linsey (eds.) (2015). Routledge International Handbook of Ignorance Studies. Londres: Routledge. <https://doi.org/10.4324/9781315867762> 
Grundmann, Reiner (2006). "Ozone and climate: scientific consensus and leadership». Scientific Technology and Human Values, 31 (1), 73-101. <https://doi.org/10.1177/0162243905280024>

- (2007). "Climate change and knowledge politics». Environmental Policy, 16 (3), 414-432. <https://doi.org/10.1080/09644010701251656>

Harré, Ron; Brockmeier, Jens y MüHlhäusler, Peter (1999). Greenspeak. A Study of Environmental Discourse. Thousand Oaks: Sage.

Hart, Herbert L.A. (1992). El concepto de derecho. Buenos Aires: Abeledo Perrot.

Howlett, Michael y Raglon, Rebecca (2001). "Constructing the environmental spectacle: green advertisements and the greening of the corporate image, 19101990. En: Fill, Alwin y MüHlhäusler, Peter (eds.). The Ecolinguistics Reader. Language, Ecology and Environment. Londres: Continuum.

Hulme, Martin (2008). «The Conquering of Climate: Discourses of fear and their Dissolution». The Geographical Journal, 147 (1), 5-16. <https://doi.org/10.1111/j.1475-4959.2008.00266.x>

Hymes, Dell H. (1986). "Models of the interaction of language and social life». En: Gumperz, John J. y Hymes, Dell H. (eds.). Sociolinguistics. Oxford: Basil Blackwell.

JaSAnOFF, Sheila (2010). «A new climate for society». Theory, Culture \& Society, 27 (2-3). <https://doi.org/10.1177/0263276409361497>

- (2011). "Cosmopolitan Knowledge: Climate Science and Global Civic Epistemology». En: Dryzek, John S.; NorgaArd, Richard B. y Schlosberg, David (eds.). The Oxford Handbook of Climate Change and Society. Oxford: University of Oxford. <https://doi.org/10.1093/oxfordhb/9780199566600.003.009>

Kempton, Willet; Boster, James S. y Hartley, Jennifer A. (1995). Environmental values in American Cultures. Cambridge (Mass.): MIT Press.

Lash, Scott; Szerszynski, Bronislaw y Wynne, Brian (eds.) (1996). Risk, Environment and Modernity: Towards a New Ecology. Londres: Sage.

LeE, Young-Ju y GreEne, Jennifer (2007). "The predict validity of an ESL placement test. A mixed method approach». Journal of Mixed Methods Research, 1 (4), 366-389.

<https://doi.org/10.1177/1558689807306148>

LeITNER, David S. (2012). «Evoking Ignorance: Abstraction and Anonymity in Social Networking's Ideals of Reciprocity». En: Hight, Casey; Kelly, Ann y Mair, Jonathan (eds.). The Anthropology of Ignorance. Nueva York: Palgrave Mcmillan, $87-118$. <https://doi.org/10.1057/9781137033123_4>

Lofstedt, Ragnar E. (1991). "Climate Change Perceptions and Energy Use Decisions in Northern Sweden». Global Environmental Change, 1, 321-4. <https://doi.org/10.1016/0959-3780(91)90058-2>

Luhmann, Niklas (1989). Ecological Communication, Cambridge: Polity Press.

- (1997). Observaciones de la modernidad. Barcelona: Paidós.

Luke, Timothy W. (1995). «On environmentality: geo-power and eco-knowledge in the discourse of contemporary environmentalism». Cultural Critique, 31, 57-81. $<$ https://doi.org/10.2307/1354445>

- (1999). «Environmentality is green governmentality». En: DARIER, Eric (ed.). Discourses of the Environment. Oxford: Blackwell, 121-51. 
Macnaghten, Phil y Szerszynki, Bronislaw (2013). «Living the global social experiment: an analysis of public discourse on solar management and its implications for governance». Global Environmental Change, 23, 465-74. <https://doi.org/10.1016/j.gloenvcha.2012.12.008>

Meira, Pablo A. (dir.) (2009). Unha aproximacio á cultural ambiental da sociedades galega. Ano 2007. Proxecto Fénix. Santiago de Compostela: Consellería de Medio Ambiente-Xunta de Galicia.

Miranda, Adolfo (2007). «Ecologismo y ficción: Acción simbólica y mirada apocalíptica en Greenpeace». Cuadernos de Información y Comunicación, 12.

Moyano, Eduardo; Paniagua, Ángel y Lafuente, Regina (2009). «Políticas ambientales, cambio climático y opinión pública en escenarios regionales. El caso de Andalucía». Revista Internacional de Sociología, 67 (3). <https://doi.org/10.3989/ris.2008.01.23>

MüHlhäUsler, Peter (2003). Language of Environment, Environment of Language. A Course in Ecolinguistics. Londres: Battlebridge.

Nisbet, Mathew C. (2009). "Communicating Climate Change: Why frames matter for public engagement». Environment, 51 (2), 2-13. $<$ https://doi.org/10.3200/ENVT.51.2.12-23>

Nisbet, Matheuw C. (2011). "Public Opinion and Participation». En: DryzeK, John S.; NorgaArd, Richard B. y Schlosberg, David (eds.). The Oxford Handbook of Climate Change and Society. Oxford: University of Oxford.

NorgaArd, Kari M. (2011a). Living in Denial: climate change, emotions, and everyday life. Cambridge (Mass.): MIT Press. <https://doi.org/10.7551/mitpress/9780262015448.001.0001>

- (2011b). «Climate Denial: Emotion, Psychology, Culture, and Political Economy». En: Dryzek, John S.; Norgand, Richard B. y Schlosberg, David (eds.). The Oxford Handbook of Climate Change and Society. Oxford: University of Oxford. <https://doi.org/10.1093/oxfordhb/9780199566600.003.0027>

O'Riordan, Timothy y JäGER, Jill (1996). Politics of climate change: A European perspective. Londres: Routledge.

Oltra, Christian y Sala, Roser (2015). «La implicació del públic en els riscos derivats de la contaminació atmosferica urbana». Papers, 100 (4), 477-492. $<$ https://doi.org/10.5565/rev/papers.2175>

Onwuegbuzie, Anthony J. y Teddlie, Charles (2003). "A Framework for Analyzing Data in Mixed Methods Research». En: Tashakkori, Abbas y Teddle, Charles (eds.) (2003). Handbook of Mixed Methods in Social \& Behavioral Research. Thousand Oaks: Sage.

Optow, Susan y Weiss, Leah (2000). «New ways of thinking about Environmentalism. Denial and the Process of Moral Exclusion in Environmental Conflict». Journal of Social Issues, 56 (3), 475-490. <https://doi.org/10.1111/0022-4537.00179>

Pettenger, Mary E. (2007). «Introduction: Power, Knowledge and the Social Construction of Climate Change». En: Pettenger, Mary E. (ed.). The Social Construction of Climate Change. Hampshire: Ashgate Pub.

Polski, Colin y Eakin, Hallie (2011). «Global Change Vulnerability Assessments: Definitions, Challenges, and Opportunities». En: Dryzek, John S.; NorgaArd, Richard B. y Schlosberg, David (eds.). The Oxford Handbook of Climate Change and Society. Oxford: University of Oxford. <https://doi.org/10.1093/oxfrodhb/9780199566600.003.0014> 
Poortinga, Wouter y Pidgeon, Nick (2003). Public Perception of Risk, Science and Governance: Main Findings of a British Survey of Five Risk Cases. Norwich: University of East Anglia.

Poortinga, Wouter; Pidgeon, Nick y Lorenzini, Irene (2006). «Public perceptions of Nuclear Power, Climate Change and Energy Options in Britain». Working Papers 0602. University of East Anglia. <https://sp.ukdataservice.ac.uk/doc/5357/mrdoc/pdf/5357userguide.pdf>.

Prades, Ana; Espluga Trenc, Josep y Horlick-Jones, Tom (2015). «Riesgos tecnológicos, conflictos sociales y políticas ambientales. Del estudio de las percepciones a la implicación pública». Papers, 100 (4), 395-423. <https://doi.org/10.5565/rev/papers.2223>

Quesada Pérez, Montse; Blanco Castilla, Elena; Teruel Rodríguez, Laura (2015). «El cambio climático en la prensa europea: discurso editorial en El País, Le Monde, The Guardian y Frankfurter Allgemeine». Estudios Sobre el Mensaje Periodístico, 21 (1), 523-539. <https://doi.org/10.5209/rev_ESMP.2015.v21.n1.49109>

Ramos Torre, Ramón (2018). «Futuros climáticos en disputa». Revista Española de Investigaciones Sociológicas, 161, 87-103. <https://doi.org/10.5477/cis/reis.161.87>

Ricoeur, Paul (1988). El discurso de la acción. Madrid: Cátedra.

Rossman, Gretchen B. y Wilson, Bruce L. (1985). «Numbers and words: Combining quantitative and qualitative methods in a single-scale evolution study». Evaluation Review, 5, 627-643. <https://doi.org/10.1177/0193841X8500900505>

Sala, Roser; Oltra, Christian; Solà, Rosario y Gamero, Nuria (2008). «Cambio climático y opinión pública en España: percepción del problema y política energética». Sistema, 205, 93-106.

Santiago Paloma (2006). «El medio ambiente en las encuestas del CIS: la sensibilidad medioambiental en España». En: Castro, R. de (coord.). Persona, sociedad y medio ambiente. Perspectiva de la investigación social de la sostenibilidad. Sevilla: Junta de Andalucía, Consejería de Medio Ambiente, 152-166.

Sarabia-Sánchez, Francisco J. y Bianchi, Enrique-Carlos (2019). "The credibility of environmental problemas in Argentina and Spain / La credibilidad de los problemas ambientales en Argentina y España». PsyEcology, 10 (3), 344-378. <https://doi.org/10.1080/21711976.2019.1602338>

Schutz, Alfred (1970). Reflections on the problem of relevance. New Haven y Londres: Yale University Press.

- (1996). Collected Papers 4. Dordrecht: Kluwer. <https://doi.org/10.1007/978-94-017-1077-0>

Schutz, Alfred y Luckmann, Thomas (1973). Las estructuras del mundo de la vida. Buenos Aires: Amorrortu.

Sloman, Steven y Fernbach, Philip (2017). Knowledge Illusion. Nueva York: Penguin Books.

Slomi-Nevo, Vered y Nevo, Isaac (2009). "Conflictive findings in mixed methods research». Journal of Mixed Methods Research, 3 (2), 109-128. <https://doi.org/10.1177/1558689808330621>

Smith, William D. (2007). «Presence of Mind as Working Climate Change Knowledge: A Totomac Cosmopolitics». En: Pettenger, Mary E. (ed.). The Social Construction of Climate Change. Hampshire: Ashgate Pub. 
Sperber, Dan y Wilson, Dreide (1994). La relevancia. Madrid: Visor.

Szerszynski, Bronislaw (1996). "On Knowing What to Do: Environmentalism and the Modern Problematic». En: Lash, C.; Szerszynski, B. y Wynne, B. (eds.). Risk, Environment and Modernity: Towards a New Ecology. Londres: Sage, 104-137. <https://doi.org/10.4135/9781446221983.n5>

- (2010). «Reading and Writing the Weather». Theory, Culture \& Society, 27 (2-3), 9-30. <https://doi.org/10.1177/0263276409361915>

Szerszynski, Bronislaw; Lash, Scott y Wynne, Brian (1996). «Introduction: Realism, Ecology and the Social Sciences». En: Lash, Scott; Szerszynski, Bronislaw y Wynne, Brian (eds.) (1996). Risk, Environment and Modernity: Towards a New Ecology. Londres: Sage, 1-25. <https://doi.org/10.4135/9781446221983.n1>

Tashakkori, Abbas y Teddlie, Charles (1998). Mixed methodology: Combining the qualitative and quantitative approaches. Thousand Oaks: Sage.

- (eds.) (2003). Handbook of Mixed Methods in Social \& Behavioral Research. Thousand Oaks: Sage.

Ueкотter, Frank y LüBKen, Uwe (2014). Managing the unknown. Essays on environmental ignorance. Nueva York y Oxford: Berghahn Books.

UNGAR, Sheldon (1992). "The rise and (relative) decline of global warming as a social problem». The Sociological Quarterly, 33 (4), 483-501. <https://doi.org/10.1111/j.1533-8525.1992.tb00139.x>

Vicente-Mariño, Miguel y Vicente-Torrico, David (2014). «Presencia y funciones del discurso científico en la cobertura informativa y cinematográfica del cambio climático». Prisma Social, 12,120-142.

Voloshinov, Valentin (1993). El marxismo y la filosofía del lenguaje. Madrid: Alianza. Wardhaugh, Ronald (1986). An introduction to sociolinguistics. Oxford: Basil Blackwell.

Wibeck, Victoria; Hansom, Anders y Anshelm, Jonas (2015). "Questioning the technological fix to climate change: lay sense-making of geoengineering in Sweden». Energy Research \& Social Science, 7, 23-30. https://doi.org/10.1016/j.erss.2015.03.001

Wibeck, Victoria; Hamson, Anders; Anshelm, Jonas et al. (2017). "Making sense of climate engineering: a focus group study of lay publics in four countries». Climate Change, $145,1$. <https://doi.org/10.1007/s10584-017-2067-0>

Witcher, Ann; Onwuegbuzie, Anthony J. y Minor, Lynn C. (2001). «Characteristics of effective teachers: Perceptions of preservice teachers». Research in the Schools, 8 (2), 45-57.

Wynne, Brian (1994). «Public understanding of science». En: Jasanoff, Sheila; Markle, Gerald E.; Petersen, James C. y Pinch, Trevor (eds.). Handbook of Science and Technologies Studies. Thousand Oaks: Sage, 361-388.

- (1996). «May the Sheep Safely Graze? A Reflexive View of the Expert-Lay Knowledge Divide». En: Lash, Scott; Szerszynski, Bronislaw y Wynne, Brian (eds.) (1996). Risk, Environment and Modernity: Towards a New Ecology. Londres: Sage, 44-83.

Zerubavel, Eviatar (1993). Fine Line. Making distinctions in every day life. Chicago: University of Chicago Press.

- (2006). The Elephant in the Room. Silence and Denial in Every Life. Oxford y Nueva York: Oxford University Press.

ZizeK, Slavoj (2001). El espinoso sujeto. El centro ausente de la ontología política. Buenos Aires: Paidós. 